\title{
Kinetic term anarchy for polynomial chaotic inflation
}

\author{
Kazunori Nakayama, ${ }^{a, b}$ Fuminobu Takahashi ${ }^{b, c}$ and Tsutomu T. Yanagida ${ }^{b}$ \\ ${ }^{a}$ Department of Physics, University of Tokyo, \\ Tokyo 113-0033, Japan \\ ${ }^{b}$ Kavli Institute for the Physics and Mathematics of the Universe (WPI), \\ Todai Institutes for Advanced Study, University of Tokyo, \\ Kashiwa 277-8583, Japan \\ ${ }^{c}$ Department of Physics, Tohoku University, \\ Sendai 980-8578, Japan \\ E-mail: kazunori@hep-th.phys.s.u-tokyo.ac.jp, \\ fumi@tuhep.phys.tohoku.ac.jp, tsutomu.tyanagida@ipmu.jp
}

ABSTRACT: We argue that there may arise a relatively flat inflaton potential over superPlanckian field values with an approximate shift symmetry, if the coefficients of the kinetic terms for many singlet scalars are subject to a certain random distribution. The inflation takes place along the flat direction with a super-Planckian length, whereas the other light directions can be stabilized by the Hubble-induced mass. The inflaton potential generically contains various shift-symmetry breaking terms, leading to a possibly large deviation of the predicted values of the spectral index and tensor-to-scalar ratio from those of the simple quadratic chaotic inflation. We revisit a polynomial chaotic inflation in supergravity as such.

KEYwORDS: Cosmology of Theories beyond the SM, Supergravity Models, Global Symmetries

ARXiv EPRINT: 1406.4265 


\section{Contents}

1 Introduction 1

2 Kinetic term anarchy for large-field inflation $\quad 2$

2.1 Model with shift symmetry at the renormalizable level 3

$\begin{array}{lll}2.2 & \text { Emergent shift symmetry } & 6\end{array}$

$\begin{array}{llr}3 & \text { Polynomial chaotic inflation } & 7\end{array}$

4 Discussion and conclusions $\quad 9$

\section{Introduction}

Inflation elegantly solves various problems of the standard big bang cosmology such as the horizon and flatness problems $[1,2]$. The slow-roll inflation paradigm [3, 4] has enjoyed a remarkable success in explaining observations of cosmic microwave background and largescale structure. Measuring the primordial B-mode polarization will be a further important step toward a proof of inflation. ${ }^{1}$

There are two important parameters, the spectral index, $n_{s}$, and the tensor-to-scalar ratio, $r$, which characterize the metric perturbations generated during inflation. The ongoing and planned B-mode experiments are targeting $r \gtrsim 10^{-2}-10^{-3}$, for which the inflaton field excursion during the last 50 or 60 e-foldings exceeds the Planck scale [6]. For inflation models with $r$ within the expected sensitivity of the foreseeable experiments, therefore, one has to have a good control of the inflaton potential over super-Planckian field ranges. This places a stringent constraint on the inflation model building. The problem becomes acute in supergravity, where the scalar potential exponentially blows up beyond the Planck scale for a general form of the Kähler potential.

In the pioneering paper by Kawasaki, Yamaguchi and one of the present authors (TTY) $[7,8]$, they proposed a simple prescription to realize a chaotic inflation model [9] in supergravity by introducing a shift symmetry

$$
\Phi \rightarrow \Phi+i \alpha,
$$

where $\alpha$ is a real transformation parameter. The Kähler potential is assumed to respect the above shift symmetry, but the superpotential contains explicit breaking terms:

$$
\begin{aligned}
K & =\frac{1}{2}\left(\Phi+\Phi^{\dagger}\right)^{2}+|X|^{2}+\cdots, \\
W & =M X \Phi
\end{aligned}
$$

\footnotetext{
${ }^{1}$ Recently the BICEP2 experiment [5] announced detection of the primordial B-mode polarization. Other observations such as Planck are necessary to confirm/refute the result.
} 
where $\Phi$ forms a Dirac mass term with another chiral superfield $X$. The imaginary component of $\Phi$ has a quadratic potential up to super-Planckian field values thanks to the shift symmetry. There are many variants and extensions of the chaotic inflation model in the same spirit. See e.g. refs. [10-18]. The inflaton potential, and therefore the predicted values of the spectral index $n_{s}$ and tensor-to-scalar ratio $r$ sensitively depend on the nature of the shift-symmetry breaking terms.

One of the central issues in large-field inflation models is the quality of the shift symmetry. If the symmetry is of high quality, the inflaton potential will be relatively flat and remain under control up to field values much greater than the Planck scale. On the other hand, if the shift symmetry is only approximate (or accidental), the inflaton potential might become extremely steep for the field values greater than a few ten times the Planck scale. In the latter case, the flatness of the inflaton potential, or the quality of the shift symmetry, might be due to the anthropic requirement that our universe experience inflation with the total e-folding number 50 or 60.

In this letter we argue that the shift symmetry required for successful large-field inflation may arise accidentally as a consequence of random-valued coefficients of the kinetic terms for many singlets, to which we refer as "kinetic term anarchy", by analogy of the neutrino mixing anarchy hypothesis [19, 20]. That is to say, if the kinetic term coefficient for one of the singlets becomes much larger than unity, the corresponding scalar potential becomes relatively flat over super-Planckian field ranges. This may happen especially if there are many singlets. In supergravity, the large kinetic term coefficient is not enough to realize large-field inflation, and one needs a certain relation between holomorphic and nonholomorphic quadratic terms. We shall consider two cases as to the origin of such relation.

Lastly, let us here mention related works in the past. It was noted in refs. [21, 22] that a large wave function factor helps to realize the slow-roll inflation. The origin of the large factor could be due to a modulus field [21] or the inflaton itself [10, 11]. The key difference of the present work from these works is that we introduce many singlet scalars with random-valued kinetic term coefficients in order to realize the large factor. Interestingly, when applied to the right-handed sneutrino chaotic inflation [23-25], such anarchic nature may partly explain the observed large neutrino mixing angles.

\section{Kinetic term anarchy for large-field inflation}

In order to see how the shift symmetry for the successful inflation could arise, let us consider two toy models. In the first model, we introduce many singlet scalars, which respect the shift symmetry for the quadratic terms in the Kähler potential, whereas it is explicitly broken by various Planck-suppressed operators in the Kähler and super-potentials. In the second model, we discuss a possibility that the shift symmetry considered in the first one arises accidentally. Throughout this letter we adopt the Planck units in which the reduced Planck mass $M_{P} \simeq 2.4 \times 10^{18} \mathrm{GeV}$ is set to be unity. 


\subsection{Model with shift symmetry at the renormalizable level}

First let us introduce $N$ singlet chiral superfield $\phi_{i}(i=1-N)$ with the following Kähler potential:

$$
K=\frac{1}{2} Z_{i j}\left(\phi_{i}+\phi_{i}^{\dagger}\right)\left(\phi_{j}+\phi_{j}^{\dagger}\right)+\cdots,
$$

where $Z_{i j}$ is a real symmetric matrix representing the kinetic term coefficients, and the sum over repeated indices is understood. We have assumed that the scalars respect the shift symmetry, ${ }^{2}$

$$
\phi_{i} \rightarrow \phi_{i}+i \alpha
$$

at the renormalizable level. Here and in what follows we assume $\phi_{i}$ has vanishing R-charges and we impose a $Z_{2}$ symmetry $\phi_{i} \rightarrow-\phi_{i}$, although most of the following argument can be straightforwardly applied to the case without the $Z_{2}$ symmetry. Note that, because of the shift symmetry, the imaginary components of $\left\{\phi_{i}\right\}$ do not appear in the quadratic terms.

The shift symmetry is assumed to be explicitly broken by various Planck-suppressed operators such as $K \supset \phi_{i} \phi_{j} \phi_{k} \phi_{\ell}, \phi_{i} \phi_{j} \phi_{k}^{\dagger} \phi_{\ell}^{\dagger}$, etc., which are collectively represented by the dots in eq. (2.1). Specifically we assume that those Planck-suppressed operators are such that each $\operatorname{Im}\left[\phi_{i}\right]$ can take values up to the Planck scale without exponential steepening of the potential, when the other scalars are set to be at the origin. This condition on the higher dimensional operators do not depend on the quadratic term coefficient $Z_{i j}$, because the imaginary components of $\left\{\phi_{i}\right\}$ appear only in the higher dimensional operators owing to the shift symmetry.

Our main idea is as follows. Let us diagonalize the kinetic term coefficients $Z_{i j}$ by an orthogonal matrix:

$$
\begin{aligned}
\phi & =R \tilde{\phi}, \\
R^{T} Z R & =\operatorname{diag}\left(z_{i}\right) \equiv D_{z}
\end{aligned}
$$

with

$$
0<z_{1} \leq z_{2} \leq \cdots \leq z_{N}
$$

where $R$ is an $N \times N$ orthogonal matrix, and we have suppressed the indices in (2.3) and (2.4). The canonically normalized fields $\left\{\Phi_{i}\right\}$ are given by

$$
\Phi=\sqrt{D_{z}} R^{T} \phi .
$$

If the kinetic term coefficient matrix $Z_{i j}$ is subject to a certain random distribution, its largest eigenvalue, $z_{N}$, could be much larger than the typical eigenvalues which we set to be of order unity. It implies that, in terms of the canonically normalized field, $\Phi_{N}=$ $\sqrt{z_{N}} \tilde{\phi}_{N}$, its imaginary component can take super-Planckian field values, $\left|\operatorname{Im}\left[\Phi_{N}\right]\right| \lesssim \sqrt{z_{N}}$.

\footnotetext{
${ }^{2}$ With an abuse of notation, we shall use the same symbol to denote both a chiral superfield and its lowest component.
} 
Therefore, such a direction is a good candidate for the inflaton. Note that, when expressed in terms of the canonically normalized field, the large eigenvalue $z_{N}$ naturally suppresses all the shift-symmetry breaking terms of $\Phi_{N}$, and the inflaton potential remains relatively flat for $\left|\operatorname{Im}\left[\Phi_{N}\right]\right| \lesssim \sqrt{z_{N}}$. In particular, the inflaton mass is suppressed by the large $z_{N}$. The inflaton field excursion depends on the tensor-to-scalar ratio $r$, and one needs typically $z_{N}$ greater than several tens for $r \gtrsim 0.01-0.1$. Thus, large eigenvalues of $Z$ help us to have a good control of the inflaton potential over super-Planckian field ranges.

The inflaton potential is lifted by shift-symmetry breaking terms. In order to account for the observed density perturbations, the typical mass scale of the inflaton is of order $10^{12-13} \mathrm{GeV}$ for $r=\mathcal{O}(0.01-0.1)$. We assume that such inflaton potential arises from the following non-renormalizable interaction in the superpotetnial,

$$
W=\kappa_{i j}\langle Q Q\rangle X_{i}\left(\phi_{j}+\cdots\right)=m_{i j} X_{i}\left(\phi_{j}+\cdots\right)
$$

where $\langle Q Q\rangle$ schematically represents a condensate of hidden quarks, and we have introduced a set of chiral superfields $X_{i}(i=1-N)$ with an $R$-charge of 2 and a negative parity under $Z_{2}$. We have defined the mass matrix $m_{i j} \equiv \kappa_{i j}\langle Q Q\rangle$, and the required inflaton mass scale can be realized for e.g. $\langle Q Q\rangle \sim\left(10^{16} \mathrm{GeV}\right)^{2}$ and $\kappa_{i j} \sim 1$. Because of the $R$-charge assignment, $\left\{\phi_{i}\right\}$ forms a Dirac mass $m_{i j}$ with $\left\{X_{j}\right\}$. The dots represent higher order terms in $\phi_{i}$ such as $\phi_{i} \phi_{j} \phi_{k}$ etc. We focus on the leading term in $\phi_{i}$ below to see the qualitative features. The effect of higher order terms will be studied in the next section.

Let us rewrite the superpotential as

$$
W=M_{i j} X_{i} \Phi_{j}
$$

where we have defined $M_{i j} \equiv m_{i j} R_{j k} / \sqrt{z_{k}}$. Note that, as mentioned before, the inflaton mass is suppressed by a factor of $\sqrt{z_{N}} \gg 1$. In the following we assume $\left\{X_{i}\right\}$ is canonically normalized. We are interested in a case where $\operatorname{Im}\left[\Phi_{N}\right]$ takes super-Planckian field values. Let us rotate $X=V \tilde{X}$ by a unitary matrix $V$ so that $\tilde{M} \equiv V^{T} M$ satisfy $\tilde{M}_{i j}=0$ for $i>j$. Focusing on the $\tilde{X}_{N}$, the superpotential looks like

$$
W=\tilde{X}_{N}\left(\tilde{M}_{N N} \Phi_{N}+\sum_{I=1}^{N-1} \tilde{M}_{N I} \Phi_{I}\right)+W_{H}\left(\tilde{X}_{I}, \Phi_{I}\right)
$$

where $W_{H}$ does not depend on $\tilde{X}_{N}$ or $\Phi_{N}$, and here and in what follows the subscript $I$ runs from 1 to $N-1$. When $\operatorname{Im}\left[\Phi_{N}\right]$ develops a super-Planckian field value, the first term in the parenthesis gives a large contribution to the $F$-term of $\tilde{X}_{N}$. For successful inflation, the $F$-term should not be absorbed by the other terms. This can be realized in either of the following two ways. First, if $\Phi_{I}$ has a mass much heavier than $\left|\tilde{M}_{N I}\right|$ from its interactions with $\tilde{X}_{I}$ in $W_{H}$, the $\Phi_{I}$ is stabilized by its large mass, and it cannot absorb the $F$-term. Secondly, if $\left|\tilde{M}_{N I}\right|<\left|\tilde{M}_{N N}\right|$, the $\Phi_{I}$ cannot absorb the $F$-term for $\left|\Phi_{I}\right| \lesssim 1$. Thus, the $F$-term of $\tilde{X}_{N}$ receives its main contribution from $\left\langle\Phi_{N}\right\rangle$, if the other scalars $\Phi_{I}$ are either heavier than $\left|\tilde{M}_{N I}\right|$ or their couplings to $\tilde{X}_{N}$ is sufficiently weak. This is tantamount to saying that $\tilde{X}_{N}$ and $\Phi_{N}$ form (more or less) degenerate mass eigenstates. 
Now let us suppose that the universe is dominated by the $F$-term of $\tilde{X}_{N}$ during inflation. Then the scalars $\Phi_{I}$ and $X_{I}$ heavier than the Hubble parameter during inflation can be integrated out. The other ones including $\tilde{X}_{N}$ generically acquire the Hubble-induced mass from the Planck-suppressed interactions, $K \supset-\left|\Phi_{I}\right|^{2}\left|\tilde{X}_{N}\right|^{2}$ and $-\left|\tilde{X}_{i}\right|^{2}\left|\tilde{X}_{N}\right|^{2}$. We therefore assume that all the scalars other than the inflaton are stabilized at the origin during inflation. ${ }^{3}$ If these conditions are satisfied, the inflaton potential is approximately given by $[7,8]$

$$
V_{\mathrm{inf}}\left(\Phi_{N}\right) \approx \frac{1}{2} M^{2} \varphi^{2}
$$

where we have defined $\varphi \equiv \sqrt{2} \operatorname{Im}\left[\Phi_{N}\right]$ and $M \equiv\left|\tilde{M}_{N N}\right|$. The above inflaton potential is valid up to $\varphi \sim \sqrt{z_{N}}$. Thus, a simple quadratic inflation can be realized because of the approximate shift symmetry with the aid of the kinetic term anarchy. Note that, in general, the inflaton condensate after inflation is composed of various mass eigenstates, because the mass eigenstates change in the course of the evolution of the inflaton. This could affect the inflaton decay process and thermal history after inflation.

So far we have simply assumed that the kinetic term coefficient matrix $Z_{i j}$ is subject to a certain random distribution so that one (or more) of the eigenvalues can happen to be much larger than unity. Let us study a concrete example if this can be indeed realized. In order to ensure the positive definite eigenvalues, we assume that $Z_{i j}$ takes the following form,

$$
Z=\frac{1}{N} Y^{\dagger} Y
$$

where $Y_{i j}$ is a complex-valued $N \times N$ non-singular matrix. If each element $Y_{i j}$ follows a complex normal distribution with the variance $\sigma=1$, the averaged eigenvalue densities of $Z$ is given by the Marcenko-Pastur law [26],

$$
f(z)=\frac{1}{2 \pi} \sqrt{\frac{4-z}{z}} \quad(z>0)
$$

in the large $N$ limit, and the averaged eigenvalue is unity. For a finite value of $N \gg 1$, the averaged largest eigenvalue $z_{N}$ is given by $\left\langle z_{N}\right\rangle \approx 4$, and its typical fluctuations is of order $N^{-2 / 3}[27,28]$. Therefore, the typical value of $z_{N}$ is not large enough to realize the large-field inflation with $r=\mathcal{O}(0.01-0.1){ }^{4}$ The probability for $z_{N}$ to take atypical large value is exponentially suppressed, but it is non-zero [29]:

$$
P\left(z_{N}\right) \sim \exp \left[-N z_{N}\right] \quad \text { for } z_{N} \gg 4 .
$$

If the kinetic term coefficient matrix $Z_{i j}$ is determined by the vacuum expectation values (VEVs) of the moduli fields, and if there are a sufficiently large number of different

\footnotetext{
${ }^{3}$ Precisely speaking, $\Phi_{I}$ develops the inflaton-dependent VEV, and there are also small mixings between $\Phi_{N}$ and $\Phi_{I}$. Also, if some of them acquire a negative Hubble-induced mass, they will be stabilized below the Planck scale and acquire a mass of order the Hubble parameter. Such deviation from the origin or the small mixings with the $\Phi_{N}$ only result in slight modification of the effective inflaton potential, and the inflaton dynamics as well as the predicted $n_{s}$ and $r$ are not significantly modified.

${ }^{4}$ The situation does not improve even if one considers a rectangular matrix for $Y_{i j}$.
} 
vacua in the string landscape, it may be possible to realize $Z_{i j}$ with $z_{N}$ greater than several tens, which support large-field inflation. Such selection of $Z_{i j}$ may be justified by anthropic arguments.

We may consider another distribution for $Z_{i j}$. Indeed, there is no a priori reason to assume that $Z_{i j}$ is given by the square of random matrices like (2.11). If the random nature of $Z_{i j}$ reflects complicated moduli stabilization dynamics, it is not unlikely that it involves some non-trivial dependence on the random matrices. For instance it will be more likely to realize $z_{N} \gg 1$ if the distribution is proportional to $\exp \left[Y^{\dagger} Y / N\right]$. Alternatively, it is also possible that $Z$ involves the inverse of the random matrices. In either case, the largest eigenvalue $z_{N}$ can be easily much larger than unity, even if the typical eigenvalues are of order unity.

The number of singlets is also likely to have an important effect on how large $z_{N}$ can be, although it is difficult to estimate it quantitatively because of our ignorance of the UV theory. Our main point is that, if $Z_{i j}$ is subject to a certain random distribution, the kinetic term coefficient for one of the singlets could happen to be enhanced, which helps to realize a flat potential over super-Planckian field ranges suitable for large-field inflation.

\subsection{Emergent shift symmetry}

Now we go on to the second model. In the previous case we have assumed the shift symmetry for the quadratic term in the Kähler potential. Here we discuss a possibility that such shift symmetry arises from the anarchic nature of both holomorphic and nonholomorphic quadratic couplings of the singlets.

First of all, let us note that, in global supersymmetry, the holomorphic quadratic terms do not contribute to the scalar potential, and therefore, the enhancement of the kinetic term coefficient suffices for the flat potential over super-Planckian ranges. In supergravity, on the other hand, the existence of the holomorphic terms in the Kähler potential is crucial for the inflaton to take super-Planckian field values. This is because of the exponential factor in the supergravity scalar potential; the inflaton component should not appear in the Kähler potential with unsuppressed coefficients.

Let us consider the Kähler potential for the singlet scalars $\left\{\phi_{i}\right\}(i=1-N)$, which generically contain both holomorphic and non-holomorphic quadratic couplings like $\phi_{i} \phi_{j}+$ h.c. and $\phi_{i}^{\dagger} \phi_{j}$. Here we rearrange the Kähler potential in such a way that anarchic nature of those two kinds of coupling coefficients can be treated in a unified manner:

$$
K=\frac{1}{2} Z_{a b} \varphi_{a} \varphi_{b}+\cdots,
$$

where $Z_{a b}$ is a real symmetric $2 N \times 2 N$ matrix, and we have defined

$$
\varphi_{a} \equiv\left\{\begin{array}{ll}
-i\left(\phi_{j}-\phi_{j}^{\dagger}\right) & \text { for } a=j \\
\phi_{j}+\phi_{j}^{\dagger} & \text { for } a=N+j
\end{array},\right.
$$

where $a$ and $j$ run from 1 to $2 N$ and 1 to $N$, respectively. We assume that various Plancksuppressed operators shown by the dots in (2.14) are such that, if the eigenvalues of $Z_{a b}$ 
were of order unity, the scalar potential would remain relatively flat along each $\varphi_{a}$ up to the Planck scale when the other scalars are set to be at the origin.

Let us diagonalize $Z_{a b}$ as

$$
\begin{aligned}
\varphi_{a} & =R \tilde{\varphi}_{a} \\
R^{T} Z R & =\operatorname{diag}\left(z_{a}\right) \equiv D_{Z}
\end{aligned}
$$

with $z_{1} \leq \cdots \leq z_{2 N}$, where $R$ is a $2 N \times 2 N$ orthogonal matrix. Suppose that $Z_{a b}$ follows a certain random distribution and that the largest eigenvalue $z_{2 N}$ happens to be much larger than unity, while the others are of order unity. ${ }^{5}$ Focusing on this direction, the Kähler potential

$$
K=\frac{z_{2 N}}{2}\left(\tilde{\phi}_{2 N}+\tilde{\phi}_{2 N}^{\dagger}\right)^{2}+\frac{1}{2}\left(\tilde{\phi}_{2 N}-\tilde{\phi}_{2 N}^{\dagger}\right)^{2}+\cdots,
$$

where we have defined $\varphi_{2 N} \equiv \tilde{\phi}_{2 N}+\tilde{\phi}_{2 N}^{\dagger}$ and dropped a numerical coefficient of order unity in the second term for simplicity, and the dots represent the other quadratic terms as well as higher order terms with a coefficient of order unity. Note that the imaginary component of $\tilde{\phi}_{2 N}$ is given by a certain combination of $\phi_{i}+\phi_{i}^{\dagger}$ and $\phi_{i}-\phi_{i}^{\dagger}$. The above Kähler potential exhibits an approximate shift symmetry along the imaginary component of $\tilde{\phi}_{2 N}$.

The kinetic term coefficient of both the real and imaginary components of $\tilde{\phi}_{2 N}$ is roughly given by $z_{2 N} \gg 1$, which suppresses the reaction of these components to the potential term and effectively enhances the friction on the motion. This would lead to the inflation if the potential remains relatively flat up to the Planck scale. However, the scalar potential for the real component of $\tilde{\phi}_{2 N}$ becomes exponentially steep at subPlanckian values because of the enhanced kinetic term coefficient. On the other hand, the imaginary component, $\operatorname{Im}\left[\tilde{\phi}_{2 N}\right]$, can take a value close to the Planck scale without exponential steepening, because, even though their kinetic term coefficient is large, they do not appear in the Kähler potential with an enhanced coupling. That is why the chaotic inflation in supergravity is possible in the presence of a large kinetic term coefficient with the (approximate) shift symmetry.

One can see this in a more conventional language by making $\tilde{\phi}_{2 N}$ canonically normalized:

$$
\Phi_{2 N} \equiv \sqrt{z_{2 N}} \tilde{\phi}_{2 N}
$$

Then, the imaginary component of $\Phi_{2 N}$ can take a super-Planckian field value up to $\left|\operatorname{Im}\left[\Phi_{2 N}\right]\right| \lesssim \sqrt{z_{2 N}}$, which realizes the chaotic inflation.

\section{Polynomial chaotic inflation}

Now let us consider concrete models. If the shift symmetry is accidental (or emergent), there are generically various shift-symmetry breaking terms, which can modify the inflaton

\footnotetext{
${ }^{5}$ This condition sets a non-trivial relation between the holomorphic and non-holomorphic coupling coefficients of the quadratic terms in the basis of $\left\{\phi_{i}\right\}$.
} 
potential from the simple quadratic potential. Therefore, we expect that the inflaton potential can be approximated by polynomials in the inflaton. We revisit the polynomial chaotic inflation in supergravity proposed by the present authors as such $[15,16]{ }^{6}$ The Kähler and superpotential for $\phi_{i}$ and $X_{i}(i=1-N)$ are

$$
\begin{aligned}
K & =\frac{1}{2} Z_{i j}\left(\phi_{i}+\phi_{i}^{\dagger}\right)\left(\phi_{j}+\phi_{j}^{\dagger}\right)+\left|X_{i}\right|^{2}+K_{N R}, \\
W & =X_{i}\left(m_{i j} \phi_{i j}+\lambda_{i j k \ell} \phi_{j} \phi_{k} \phi_{\ell}+\cdots\right)
\end{aligned}
$$

where $\phi_{i}$ respects the shift symmetry on the quadratic terms, and $K_{N R}$ denotes higher order terms in the Kähler potential, which include terms like $X_{i} X_{j} X_{k}^{\dagger} X_{\ell}^{\dagger}$ as well as shiftsymmetry breaking terms such as $\phi_{i} \phi_{j} \phi_{k} \phi_{\ell}, \phi_{i} \phi_{j} \phi_{k}^{\dagger} \phi_{\ell}^{\dagger}$, etc. with coefficients of $O(1)$. We assume that $m_{i j}$ and $\lambda_{i j k \ell}$ are the same order in the Planck unit (cf. eq. (2.7)) and higherorder terms with coefficients of the same order are represented by the dots. We have imposed $Z_{2}$ symmetry under which $\phi_{i} \rightarrow-\phi_{i}, X \rightarrow-X$ and assigned $\mathrm{U}(1)_{R}$ charges as $R\left(\phi_{i}\right)=0$ and $R\left(X_{i}\right)=2$.

After diagonalizing the kinetic term by using $\Phi=\sqrt{D_{z}} R^{T} \phi$, we obtain

$$
\begin{aligned}
K & =\frac{1}{2}\left(\Phi_{i}+\Phi_{i}^{\dagger}\right)^{2}+|X|^{2}+K_{N R}, \\
W & =X_{i}\left(\tilde{m}_{i j} \Phi_{i}+\tilde{\lambda}_{i j k \ell} \Phi_{j} \Phi_{k} \Phi_{\ell}+\cdots\right),
\end{aligned}
$$

where $\tilde{m}_{i j}=m_{i k} R_{k j} / \sqrt{z_{j}}$ and $\tilde{\lambda}_{i j k \ell}=\lambda_{i a b c} R_{a j} R_{b k} R_{c \ell} / \sqrt{z_{j} z_{k} z_{\ell}}$. As described in section 2, the imaginary component of $\Phi_{N}$ can be regarded as the inflaton, because the scalar potential does not exponentially blow up for $\left|\operatorname{Im}\left[\Phi_{N}\right]\right| \lesssim \sqrt{z_{N}}$. The relevant terms including $\Phi_{N}$ can be rewritten as

$$
W_{\mathrm{eff}} \simeq X_{N}^{(1)} M_{N N} \Phi_{N}+X_{N}^{(2)} \tilde{\lambda}_{N N N N} \Phi_{N}^{3}+\cdots,
$$

where we have defined $X^{(1)}=U X$ and $X^{(2)}=V X$ for some unitary matrices $U$ and $V$ such that $U_{j i} \tilde{m}_{j N}=0$ and $V_{j i} \tilde{\lambda}_{j N N N}=0$ for $i<N$. We assume that the other fields are either sufficiently heavy or their couplings to $X^{(1)}$ and $X^{(2)}$ are sufficiently weak so that the $F$-terms of $X_{N}^{(1)}$ and $X_{N}^{(2)}$ mainly come from the $\mathrm{VEV}$ of $\Phi_{N}$. If $X_{N}^{(2)}$ can be expressed in terms of only $X_{I}^{(1)}(I=1-N-1)$, the $F$-term potentials of $X_{N}^{(1)}$ and $X_{N}^{(2)}$ induce the quadratic and hexic potentials, respectively. In general, $X_{N}^{(1)}$ and $X_{N}^{(2)}$ are not independent from each other, and their overlap generates the quartic potential as a cross term. Therefore, the resultant inflaton potential generically takes the polynomial form. ${ }^{7}$

Let us consider a limiting case of $X_{N}^{(1)}=X_{N}^{(2)}$. Then, the relevant Kähler and superpotentials are given by

$$
\begin{aligned}
K & =\sum_{i} \frac{1}{2}\left(\Phi_{i}+\Phi_{i}^{\dagger}\right)^{2}+|X|^{2}+K_{N R}, \\
W & =X\left(M \Phi+\lambda \Phi^{3}+\ldots\right),
\end{aligned}
$$

\footnotetext{
${ }^{6}$ The polynomial chaotic inflation in supergravity was also studied in refs. [18, 30] after BICEP2.

${ }^{7}$ Even if the other scalars are not stabilized at the origin but somewhere below the Planck scale, the effective inflaton potential can be well approximated by the polynomial potential with slightly different numerical coefficients.
} 
where we have defined $X=X_{N}^{(1)}, \Phi=\Phi_{N}, M \equiv M_{N N}$ and $\lambda \equiv \lambda_{N N N N}$, and we expect $|\lambda| \sim|M| / z_{N}$ if $m_{i j} \sim \lambda_{i j k \ell}$ (see eq. (2.7)). This is exactly the same form as the polynomial chaotic inflation proposed in refs. $[15,16]$. The inflaton potential is given by

$$
V \simeq \frac{1}{2}|M|^{2} \varphi^{2}\left(1-\frac{|\lambda| \cos \theta}{|M|} \varphi^{2}+\frac{|\lambda|^{2}}{4|M|^{2}} \varphi^{4}\right)
$$

where we have defined $\varphi \equiv \operatorname{Im}[\Phi]$ and $\theta \equiv \arg [\lambda / M]$. Because of the largeness of $z_{N}$, higher order shift symmetry breaking terms in the Kähler potential are suppressed which enables successful inflation. For example, the terms quartic in the inflaton like $K_{N R} \propto|\Phi|^{4}$ are accompanied with a suppression factor $\sim 1 / z_{N}^{2}$, which induces a correction to the inflaton potential of order $H_{\mathrm{inf}}^{2} \varphi^{4} / z_{N}^{2}$. The correction to the inflaton mass remains sufficiently small for $\varphi \lesssim \sqrt{z_{N}}$.

The potential is naturally given by the polynomial and its starts to deviate from the quadratic potential at $\varphi \sim \sqrt{|M / \lambda|} \sim \sqrt{z_{N}}$, which may be close to the point above which various other corrections become important. If $M_{N N}$ is slightly smaller than $\lambda_{N N N N}$, there will be a field range where the inflaton potential is well approximated by the above polynomial inflation. The prediction on $n_{s}$ and $r$ can thus be significantly deviated from those of the chaotic inflation with a monomial potential. Importantly, as emphasized in refs. $[15,16]$, they cover the entire range allowed by the Planck results.

\section{Discussion and conclusions}

So far we have imposed the $Z_{2}$ symmetry on the scalars to simplify the analysis. In fact, such $Z_{2}$ symmetry suppresses non-thermal gravitino production from the inflaton decay [31-36], avoiding tight cosmological constraints [37]. If the $Z_{2}$ symmetry remains unbroken, we need to assign the $Z_{2}$ charge on the standard model particles and their superpartners for successful reheating. Interestingly, the $Z_{2}$ symmetry can be identified with the matter parity, which forbids baryon- and lepton-number violating operators dangerous for too fast proton decays. Then the singlet scalars play the role of right-handed sneutrinos [25]. In particular, if we apply the kinetic anarchy to the right-handed sneutrino inflation, the shift symmetry may arise naturally [38]. Let us briefly discuss this case below.

Let us consider the model (2.1)-(2.6). Instead of introducing $\left\{X_{i}\right\}$, we consider the following superpotential for $\left\{\phi_{i}\right\}$ :

$$
W=\frac{1}{2} m_{i j} \phi_{i} \phi_{j}
$$

where we do not impose $\mathrm{U}(1)_{R}$ symmetry. The $\tilde{\phi}_{N}$ tends to be the lightest one because of the large kinetic term coefficient. Then, after integrating out heavy scalars, the lightest one forms either a Majorana mass with itself or a Dirac mass with a partner, which we denote $X$. The successful inflation is possible in the latter case. Interestingly, this scenario provides a unified picture of $\Phi$ and $X$ in the original model (1.3), because both are originated from the singlet scalars $\left\{\phi_{i}\right\}$, and it is simply the eigenvalue $z_{N} \gg 1$ that makes the shift symmetry of $\Phi$ of better quality. The fact that only $\Phi$ has a shift symmetry of better quality is 
crucial for successful inflation, because, if the shift symmetry on $X$ is a good symmetry, the inflation path is destabilized by the appearance of a tachyonic direction.

Another interesting aspect of this model is that the inflaton as well as other scalars have neutrino yukawa couplings like

$$
W=y_{\alpha i} \phi_{i} L_{\alpha} H_{u}=Y_{\alpha i} \Phi_{i} L_{\alpha} H_{u},
$$

where $H_{u}$ is the up-type Higgs doublet and $L_{\alpha}$ denotes the lepton doublet with $\alpha=1,2,3$ being the generation index and $Y_{\alpha i}=y_{\alpha j} R_{j i} / \sqrt{z_{i}}$. Since the inflaton mass scale is $\sim$ $10^{13} \mathrm{GeV}$, it explains the tiny neutrino masses observed by neutrino oscillation experiments through the seesaw mechanism [39-42] for $y_{\alpha} \sim O(0.1) .{ }^{8}$ The observed large neutrino mixings may be (partly) originated from the anarchic nature of the singlet scalars including the inflaton, which nicely fits with the neutrino mass anarchy hypothesis [19, 20].

In this letter we have proposed a scenario in which an approximate shift symmetry required for large-field inflation appears because of the enhanced kinetic term coefficient. Such an enhancement may be realized if there are many singlets whose kinetic term coefficients follow some random distribution. Although the probability for such an enhancement to take place is exponentially suppressed in the simplest model, such fine-tuning may be justified by the anthropic arguments. Alternatively, if the kinetic term coefficients follow a different distribution such as the exponential or inverse of the random matrix, it becomes much easier to obtain the required enhancement. In supergravity, such an enhancement is not sufficient for successful large-field inflation, and a certain relation is required between the holomorphic and non-holomorphic quadratic terms of the inflaton. We have studied two cases in which such a relation is satisfied because of the approximate shift symmetry or the relation emerges by accident. In our scenario, the shift symmetry is only approximate, and so, the inflaton potential generically receives various shift-symmetry breaking terms. We have shown that the resultant inflaton dynamics is well approximated by the polynomial chaotic inflation. Interestingly, the predicted values of $n_{s}$ and $r$ can be significantly deviated from those of the simple quadratic chaotic inflation. In fact, it was shown in refs. $[15,16]$ that the predicted $\left(n_{s}, r\right)$ of the polynomial chaotic inflation can cover the entire ranges allowed by the Planck data. The future B-mode experiments, therefore, will be able to refute or support our inflationary scenario.

\section{Acknowledgments}

We thank Hitoshi Murayama for fruitful discussion. TTY learned that Ken-iti Izawa is working on a similar subject. This work was supported by the Grant-in-Aid for Young Scientists (B) (No. 26800121 [KN], No.24740135 [FT]), Scientific Research on Innovative Areas (No.23104008 [FT]), and Scientific Research (B) (No.26287039 [FT and TTY]), by Inoue Foundation for Science $[\mathrm{FT}]$, and by World Premier International Center Initiative (WPI Program), MEXT, Japan.

\footnotetext{
${ }^{8}$ Note that the yukawa couplings (4.2) stabilize $L_{\alpha}$ and $H_{u}$ during inflation at the origin, since both $X$ and $\Phi$ have large yukawa couplings. In ref. [16], it was assumed that only either $X$ or $\Phi$ has a renormalizable yukawa coupling $y$ and derived a constraint as $y \lesssim 10^{-5}$ for successful inflation. This constraint can avoided in the present model because both $X$ and $\Phi$ have yukawa couplings.
} 
Open Access. This article is distributed under the terms of the Creative Commons Attribution License (CC-BY 4.0), which permits any use, distribution and reproduction in any medium, provided the original author(s) and source are credited.

\section{References}

[1] A.H. Guth, The inflationary universe: a possible solution to the horizon and flatness problems, Phys. Rev. D 23 (1981) 347 [InSPIRE].

[2] D. Kazanas, Dynamics of the universe and spontaneous symmetry breaking, Astrophys. J. 241 (1980) L59 [INSPIRE].

[3] A.D. Linde, A new inflationary universe scenario: a possible solution of the horizon, flatness, homogeneity, isotropy and primordial monopole problems, Phys. Lett. B 108 (1982) 389 [INSPIRE].

[4] A. Albrecht and P.J. Steinhardt, Cosmology for grand unified theories with radiatively induced symmetry breaking, Phys. Rev. Lett. 48 (1982) 1220 [INSPIRE].

[5] BICEP2 collaboration, P.A.R. Ade et al., Detection of B-mode polarization at degree angular scales by BICEP2, Phys. Rev. Lett. 112 (2014) 241101 [arXiv: 1403.3985] [INSPIRE].

[6] D.H. Lyth, What would we learn by detecting a gravitational wave signal in the cosmic microwave background anisotropy?, Phys. Rev. Lett. 78 (1997) 1861 [hep-ph/9606387] [INSPIRE].

[7] M. Kawasaki, M. Yamaguchi and T. Yanagida, Natural chaotic inflation in supergravity, Phys. Rev. Lett. 85 (2000) 3572 [hep-ph/0004243] [INSPIRE].

[8] M. Kawasaki, M. Yamaguchi and T. Yanagida, Natural chaotic inflation in supergravity and leptogenesis, Phys. Rev. D 63 (2001) 103514 [hep-ph/0011104] [InSPIRE].

[9] A.D. Linde, Chaotic inflation, Phys. Lett. B 129 (1983) 177 [InSPIRE].

[10] F. Takahashi, Linear inflation from running kinetic term in supergravity, Phys. Lett. B 693 (2010) 140 [arXiv: 1006.2801] [INSPIRE].

[11] K. Nakayama and F. Takahashi, Running kinetic inflation, JCAP 11 (2010) 009 [arXiv: 1008.2956] [INSPIRE].

[12] R. Kallosh and A. Linde, New models of chaotic inflation in supergravity, JCAP 11 (2010) 011 [arXiv: 1008.3375] [INSPIRE].

[13] R. Kallosh, A. Linde and T. Rube, General inflaton potentials in supergravity, Phys. Rev. D 83 (2011) 043507 [arXiv: 1011.5945] [INSPIRE].

[14] K. Harigaya, M. Ibe, K. Schmitz and T.T. Yanagida, Chaotic inflation with a fractional power-law potential in strongly coupled gauge theories, Phys. Lett. B 720 (2013) 125 [arXiv:1211.6241] [INSPIRE].

[15] K. Nakayama, F. Takahashi and T.T. Yanagida, Polynomial chaotic inflation in the Planck era, Phys. Lett. B 725 (2013) 111 [arXiv:1303.7315] [INSPIRE].

[16] K. Nakayama, F. Takahashi and T.T. Yanagida, Polynomial chaotic inflation in supergravity, JCAP 08 (2013) 038 [arXiv: 1305.5099] [INSPIRE].

[17] K. Harigaya and T.T. Yanagida, Discovery of large scale tensor mode and chaotic inflation in supergravity, arXiv:1403.4729 [INSPIRE]. 
[18] R. Kallosh, A. Linde and A. Westphal, Chaotic inflation in supergravity after Planck and BICEP2, Phys. Rev. D 90 (2014) 023534 [arXiv: 1405.0270] [InSPIRE].

[19] L.J. Hall, H. Murayama and N. Weiner, Neutrino mass anarchy, Phys. Rev. Lett. 84 (2000) 2572 [hep-ph/9911341] [INSPIRE].

[20] N. Haba and H. Murayama, Anarchy and hierarchy, Phys. Rev. D 63 (2001) 053010 [hep-ph/0009174] [INSPIRE].

[21] S. Dimopoulos and S.D. Thomas, Discretuum versus continuum dark energy, Phys. Lett. B 573 (2003) 13 [hep-th/0307004] [INSPIRE].

[22] K.-I. Izawa and Y. Shinbara, Supersymmetric tuned inflation, arXiv:0710.1141 [INSPIRE].

[23] H. Murayama, H. Suzuki, T. Yanagida and J. Yokoyama, Chaotic inflation and baryogenesis by right-handed sneutrinos, Phys. Rev. Lett. 70 (1993) 1912 [InSPIRE].

[24] K. Nakayama, F. Takahashi and T.T. Yanagida, Chaotic inflation with right-handed sneutrinos after Planck, Phys. Lett. B 730 (2014) 24 [arXiv:1311.4253] [INSPIRE].

[25] H. Murayama, K. Nakayama, F. Takahashi and T.T. Yanagida, Sneutrino chaotic inflation and landscape, arXiv:1404.3857 [INSPIRE].

[26] V.A. Marcenko and L.A. Pastur, Distribution of eigenvalues for some sets of random matrices, Math. USSR-Sb 1 (1967) 457.

[27] I.M. Johnstone, On the distribution of the largest eigenvalue in principal components analysis, Ann. Statist. 29 (2001) 295.

[28] K. Johansson, Shape fluctuations and random matrices, Commun. Math. Phys. 209 (2000) 437 [math/9903134].

[29] S.N. Majumdar and M. Vergassola, Large deviations of the maximum eigenvalue for wishart and gaussian random matrices, Phys. Rev. Lett. 102 (2009) 060601 [arXiv:0811.2290] [INSPIRE].

[30] T. Kobayashi and O. Seto, Polynomial inflation models after BICEP2, Phys. Rev. D 89 (2014) 103524 [arXiv:1403.5055] [INSPIRE].

[31] M. Kawasaki, F. Takahashi and T.T. Yanagida, Gravitino overproduction in inflaton decay, Phys. Lett. B 638 (2006) 8 [hep-ph/0603265] [INSPIRE].

[32] M. Kawasaki, F. Takahashi and T.T. Yanagida, The gravitino-overproduction problem in inflationary universe, Phys. Rev. D 74 (2006) 043519 [hep-ph/0605297] [INSPIRE].

[33] T. Asaka, S. Nakamura and M. Yamaguchi, Gravitinos from heavy scalar decay, Phys. Rev. D 74 (2006) 023520 [hep-ph/0604132] [INSPIRE].

[34] M. Dine, R. Kitano, A. Morisse and Y. Shirman, Moduli decays and gravitinos, Phys. Rev. D 73 (2006) 123518 [hep-ph/0604140] [INSPIRE].

[35] M. Endo, F. Takahashi and T.T. Yanagida, Anomaly-induced inflaton decay and gravitino-overproduction problem, Phys. Lett. B 658 (2008) 236 [hep-ph/0701042] [INSPIRE].

[36] M. Endo, F. Takahashi and T.T. Yanagida, Inflaton decay in supergravity, Phys. Rev. D 76 (2007) 083509 [arXiv:0706.0986] [INSPIRE].

[37] K. Nakayama, F. Takahashi and T.T. Yanagida, Gravitino problem in supergravity chaotic inflation and SUSY breaking scale after BICEP2, Phys. Lett. B 734 (2014) 358 [arXiv: 1404.2472] [INSPIRE]. 
[38] H. Murayama, K. Nakayama, F. Takahashi and T.T. Yanagida, in preparation.

[39] P. Minkowski, $\mu \rightarrow$ er at a rate of one out of 1-billion muon decays?, Phys. Lett. B 67 (1977) 421 [INSPIRE].

[40] T. Yanagida, Horizontal symmetry and masses of neutrinos, in the proceedings of the Workshop on unified theory and baryon number in the universe, O. Sawada and A. Sugamoto eds., KEK, Tsukuba, Japan (1979).

[41] P. Ramond, The family group in grand unified theories, hep-ph/9809459 [INSPIRE].

[42] S.L. Glashow, The future of elementary particle physics, in Quarks and leptons, Cargèse lectures, M. Lévy et al. eds., Plenum Press, New York, U.S.A. (1980). 